\title{
Ulteriori contributi allo studio dei problemi ellittici in un angolo.
}

\author{
antonio Avantaggiati - Mario Troisi (Bari) $\left(^{*}\right)\left({ }^{* *}\right)$
}

Summary. - In [2] and [3] elliptic boundary value problems in a plane sector were investigated within certain Sobolev weight spaces $W_{s_{1}, s_{2}}^{r}$ and $W_{s_{1}, s_{3}}^{* r}$ (see [1]), with the exception of some well-defined values of $s_{1}-r+1$ and $s_{2}-r+1$. This paper is concerned with such boundary value problems when $s_{1}-r+1$ or $s_{2}-r+1$ are exceptional values.

Sia $A$ l'angolo aperto di $R^{2}$ con vertice nell'origine e delimitato dalle due semirette $\sigma_{1}$ e $\sigma_{2}$ di anomalie $\theta_{1}$ e $\theta_{2}$ rispettivamente, dove $-\pi / 2<\theta_{1}<\theta_{2}<3 \pi / 2$.

Assegniamo in $A$ un operatore differenziale lineare $A=A(x, \partial)$ di ordine $2 m$, propriamente ellittico in $\bar{A}$ e all'infinito, ed assegniamo, per $k=1,2$, un sistema $\left\{B_{j k}\right\}_{j=1}^{m}=\left\{B_{j k}(x, \partial)\right\}_{j=1}^{m}$ di $m$ operatori differenziali lineari di frontiera su $\sigma_{\bar{k}}$, con $B_{j k}$ di ordine $m_{j k}$, soddisfacenti la condizione complementare rispetto ad $A$ su $\bar{\sigma}_{k} \mathrm{e}$ all'infinito.

In [2] e [3] abbiamo studiato il problema

$$
\begin{aligned}
& A u=f \text { in } A, \\
& B_{j k} u=g_{j k} \text { su } \sigma_{k}, \quad j=1, \ldots, m, \quad k=1,2,
\end{aligned}
$$

cercando la soluzione in certi spazi di Sobolev con doppio peso, denotati con $W_{s_{1}, s_{2}}^{r}(\Lambda)$ e $W_{s_{1}, s_{2}}^{*}(\Lambda) \quad\left(r\right.$ reale $\geqslant 2 m$ e $>\sup _{j, k} m_{j k}+\frac{1}{2}, s_{1}, s_{2}$ reali e $\left.s_{1} \leqslant s_{2}\right)$, preliminarmente studiati in [1], dove i pesi hanno il ruolo di prescrivere il comportamento della soluzione e delle sue derivate nel vertice dell'angolo e all'infinito.

Abbiamo provato che, sotto opportune ipotesi sui coefficienti di $A$ e $B_{j k}$, esistono due sottoinsiemi $\Sigma_{1}$ e $\Sigma_{2}$ di $R$, discreti e numerabili, tali che se $s_{i}-r+1 \notin \Sigma_{i}, i=1,2$, l'operatore

$$
\mathcal{A}_{r, s_{1}, s_{a}}: u \rightarrow\left(A u,\left\{\left.B_{j k} u\right|_{\sigma_{k}}\right\}\right),
$$

definito in $W_{s_{1}, s_{2}}^{r}(\Lambda)$ ed a valori in $E_{r, s_{1}, s_{3}}(\Lambda)=W_{s_{1}, s_{2}}^{r-2 m}(\Lambda) \times \prod_{j=1}^{m} \prod_{k=1}^{2} W_{s_{1}, s_{2}}^{r-m_{j k}-\frac{1}{2}}\left(\sigma_{k}^{*}\right)$, è un operatore a indice, cioè ha nucleo di dimensione finita ed ha immagine chiusa e di codimensione finita. Inoltre i numeri $\operatorname{dim} \operatorname{Ker}\left(\mathcal{A}_{r, s_{1}, s_{\mathrm{s}}}\right), \operatorname{codim} \operatorname{Im}\left(\mathcal{A}_{r, s_{1}, s_{1}}\right)$ e indice

(*) Entrata in Redazione il 20 giugno 1973.

(**) Lavoro eseguito con contributo del C.N.R. nell'ambito del Gruppo Nazionale per I'Analisi Funzionale e le sue Applicazioni. 
$\left(\mathcal{A}_{r, s_{1}, s_{\mathrm{g}}}\right)$ risultano costanti al variare di $r, s_{1}, s_{2}$ in modo che $s_{i}-r+1, i=1,2$, appartenga ad un intervallo disgiunto da $\Sigma_{i}$.

Ricordiamo anche che $\Sigma_{i}, i=1,2$, è la proiezione su $R$ dell'insieme degli zeri di una funzione intera $g_{i}(z)$ nel piano complesso, e precisamente della funzione discriminante (cfr. il $\mathrm{n} .4 \mathrm{di}[2])$ associata a $\left(A^{\prime}\left(x^{i}, \partial\right),\left\{B_{j k}^{\prime}\left(x^{i}, \partial\right)\right\}\right)$, dove $x^{1}=0, x^{2}=\infty$ e l'apice sta a denotare la parte principale degli operatori.

Abbiamo poi provato che, sotto ulteriori ipotesi sui coefficienti di $A$ e $B_{j l_{0}}$ e se i dati $f$ e $g_{j k}$ soddisfano un numero finito (eventualmente nullo) di condizioni di compatibilità nell'origine, il problema (1) con $u \in W_{s_{1}, s_{2}}^{*_{r}}(\Lambda)$ è equivalente (in un senso precisato al n. $6 \mathrm{di}[3]$ ) al problema (1) con $u \in W_{s_{1}, s_{2}}^{r}(A)$ e con altri termini noti opportunamente collegati a $f$ e $g_{j k}$.

Ci proponiamo ora, in questa Nota, di studiare i problemi sopra considerati nel caso in cui uno dei due numeri $s_{1}-r+1$ e $s_{2}-r+1$ sia un valore eccezionale escluso dalla trattazione di [2] e [3], cioè nel caso in cui

$$
\left(s_{1}-r+1, s_{2}-r+1\right) \notin\left(R \backslash \Sigma_{1}\right) \times\left(R \backslash \Sigma_{2}\right) .
$$

Siccome, per quanto sopra accennato, lo studio del problema (1) con $u \in W_{s_{1}, s_{2}}^{*_{r}}(\Lambda)$ si può ricondurre allo studio del problema (1) con $u \in W_{s_{1}, s_{2}}^{r}(\Lambda)$, ci limiteremo a considerare soltanto quest'ultimo.

Nel caso in cui $A$ e $B_{j k}$ siano operatori omogenei ed a coefficienti costanti, indicheremo con $g=\operatorname{det} G$ la funzione discriminante associata a $\left(A,\left\{B_{j k}\right\}\right)$ e con $\Sigma_{g}$ l'insieme $\Sigma_{1}=\Sigma_{2}$ costituito dalle parti reali degli zeri di $g$.

Dimostreremo i seguenti teoremi:

TEorema I ( $\left.{ }^{1}\right)$. - Supponiamo che gli operatori $A, B_{j}$ siano omogenei ed a coefficienti costanti ed assegniamo due numeri reali $a, b$ tali che $a<s_{1}-r+1 \leqslant s_{2}-r+1<b$ e $] a, b\left[\cap \Sigma_{g}=\left[s_{1}-r+1, s_{2}-r+1\right] \cap \Sigma_{g}\right.$. Allora per ogni $u \in W_{s_{1}, s_{2}}^{r}(\Lambda)$ e tale che $\left(^{2}\right)$ $\mathcal{A}_{x, s_{1}, s_{2}} u \in F_{a+2 m}^{b+2 m}\left(\Lambda_{0}\right) \times \prod_{j=1}^{m} \prod_{k=1}^{2} F_{a+m s_{s k}}^{b+m_{j k}}\left(\sigma_{k}\right)$ si hat $u \in F_{a}^{b}\left(\Lambda_{0}\right)$.

ThoReMa II. - Se A, $B_{j k}$ sono operatori omogenei ed a coefficienti costanti e se uno dei due numeri $s_{1}-r+1$ e $s_{2}-r+1$ appartiene a $\Sigma_{g}$ e non è zero reale di g appartenente all'insieme $\{-2 m+2, \ldots, 0\}\left(^{8}\right)$, allora $\mathcal{A}_{r, s_{1}, s_{2}}$ ha immagine non chusa in $E_{r, s_{1}, s_{2}}(\Lambda)$.

(1) Osserviamo che, quando entrambi i numeri $s_{1}-r+1$ e $s_{2}-r+1$ non appartengono a $\Sigma_{g}$, la tesi di questo teorema è un'ovvia conseguenza della prop. 4.8 e del teorema 1.1 di [2]. Perciò dovremo dimostrare il teorema, solo nel caso in cui uno almeno dei due numeri $s_{1}-r+1$ e $s_{2}-r+1$ appartenga a $\Sigma_{g}$.

(2) Per tutte le notazioni che in questo lavoro adoperiamo senza definizione rinviamo a [1] e [2].

(3) I valori di $\{-2 m+2, \ldots, 0\}$ che sono zeri di $g$ possono far eccezione, nel senso che vi sono problemi per i quali $\mathcal{A}_{r, s_{1}, s_{2}}$ ha immagine chinsa anche se uno dei due numeri $s_{1}-r+1$ e $s_{2}-r+1$ è uno zero di $g$ appartenente all'insieme $\{-2 m+2, \ldots, 0\}$. Tale è, ad es., il caso del problema di Dirichlet per l'operatore di Laplace (cfr. l'esempio 1 al n. 8 di [3]). 
TeоRema III. - Supponiamo ohe gli operatori $A, B_{j k}$ abbiano coefficienti variabili soddisfacenti le ipotesi del teorema $1.1 d i[3]$ e che, per un $i \in\{1,2\}, s_{i}-r+1$ appartenga a $\Sigma_{i}$ e non sia uno zero reale di $g_{i}$ appartenente all'insieme $\{-2 m+2, \ldots, 0\}$.

Allora la (1.14) di [3] non pù̀ sussistere con $\varepsilon>0$, per cui o il nucleo $d i \mathscr{A}_{r, s_{1}, s_{2}}$ non ha dimensione finita oppure t'immagine $d i t_{r, s_{1}, s_{2}}$ non è chizsa in $E_{r, s_{1}, s_{2}}(\Lambda)$.

Le dimostrazioni dei teoremi I e II saranno date al n. 1 e la dimostrazione del teorema III sarà data al n. 3. Nel corso di tali dimostrazioni ci riferiremo a [1.], [2] e [3] sia per le notazioni sia per le tecniche usate.

Le considerazioni tenute al $n$. 1 ei danno inoltre la possibilità di provare il seguente teorema, non contenuto nei risultati di [1], [2] e [3]:

Teorema IV. - Supponiamo ehe gli operatori $A, B_{j k}$ siano omogenei ed a coefficienti costanti, che risulti $s_{1}<s_{2}\left({ }^{4}\right), s_{i}-r+1 \notin \Sigma_{g}$ per $i=1,2$ e che ogni zero che la funzione discriminante $g$ ha nella striscia $\sigma\left(s_{1}-r+1, s_{2}-r+1\right)$ non appartenga all'insieme $\left.\{-2 m+2, \ldots, 0\}{ }^{5}\right)$.

Allora l'indiee di $\mathcal{A}_{r, s_{1}, s_{2}}$ è uguale all'opposto del numero degli zeri che g ha nella striscia $\sigma\left(s_{1}-r+1, s_{2}-r+1\right)$ ciascuno contato tante volte quanto il suo ordine di molteplioità.

La dimostrazione di questo teorema sarà data al n. 2.

\section{1. - Dimostrazione dei teoremi I e II.}

Nel corso di queste dimostrazioni faremo frequentemente riferimento a [2], e per uniformarci alla trattazione di tale lavoro converremo di denotare con $L=L(\partial)$ e $P_{j k}=P_{j k}(\partial)$ gli operatori omogenei ed a coefficienti costanti $A$ \& $B_{j k}$ rispettivamente. $\mathcal{A}_{r, s_{1}, s_{2}}$ denoterà allora l'operatore definito dalla (1.4) di [2].

Per semplicità, ei limiteremo a dimostrare i teoremi I e II mettendoci nelle seguenti condizioni:

x) la funzione $g$ ha una sola radice $z_{1}$ avente parte reale uguale a $s_{1}-r+1$, e tale radice è semplice;

$$
\text { B) risulta }\left[s_{1}-r+1, s_{\mathrm{a}}-r+1\right] \cap \Sigma_{g}=\left\{s_{1}-r+1\right\} \text {. }
$$

Apparirà poi evidente quali siano le modifiche da apportare ai ragionamenti che terremo per ottenere le dimostrazioni nel caso generale, ove nel caso del teorema II si tenga anche conto del lemma 2.1.

$\left(^{4}\right)$ Teniamo presente (cfr. il teorema 1.1 di $[2]$ ) che se $s_{1} \leqslant s_{2}$ e $\left[s_{1}-r+1, s_{2}-r+1\right] \cap$ $\cap \Sigma_{g}=\phi$, allora $\mathcal{A}_{r, s_{1}, s_{\mathrm{z}}} \grave{\mathrm{e}}$ un isomorfismo algebrico e topologico surgettivo.

$\left.{ }^{5}\right)$ Come nel caso del teorema II, va anche qui rilevato che gli zeri di $g$ appartenenti all'insieme $\{-2 m+2, \ldots, 0\}$ possono far eccezione (efr. ancora l'esempio 1 del n. 8 di [3] relativo al problema di Dirichlet per l'operatore di Laplace). 
Fissiamo allora $\mathrm{i}$ numeri reali $a, b, s_{1}^{0}$ in modo che

$$
\begin{aligned}
& s_{1}^{0}<s_{1}, \quad\left[s_{1}^{0}-r+1, s_{2}-r+1\right] \cap \Sigma_{g}=\left\{s_{1}-r+1\right\} \\
& ] a, b\left[\cap \Sigma_{g}=\left\{s_{1}-r+1\right\}, \quad\right] a, b\left[\supset\left[s_{1}^{0}-r+1, s_{2}-r+1\right] .\right.
\end{aligned}
$$

Poniamo quindi $\mathfrak{F}=F_{a+2 m}^{b+2 m}\left(\Lambda_{0}\right) \times \prod_{j=1}^{m} \prod_{k=1}^{2} F_{a+m_{j_{k}}}^{b+m_{k j}}\left(\sigma_{k}\right)$, dove $\Lambda_{0}=\bar{A} \backslash\{0\}$, e ricordiamo che per il modo in eui sono stati fissati $a$ e $b$ si ha

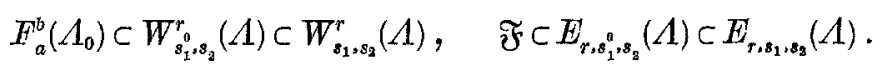

Indichiamo inoltre con $\mathcal{A}\left(F_{a}^{b}\left(\boldsymbol{\Lambda}_{0}\right)\right)$ l'immagine della restrizione di $\mathcal{A}_{r, s_{1}, s_{2}}$ al sottospazio $F_{a}^{b}\left(\Lambda_{0}\right)$.

Si riconosce facilmente, tenuto conto della densità di $F_{a}^{b}\left(\Lambda_{0}\right)$ in $W_{s_{1}, s_{2}}^{r}(\Lambda)$ e in $W_{s_{1}, s_{2}}^{r}(\Lambda)$, che $\mathfrak{F}$ è denso in $E_{r, s_{1}^{\circ}, s_{2}}(\Lambda)$ e in $E_{r, s_{1}, s_{2}}(\Lambda)$ e che $\mathcal{A}\left(H_{a}^{b b}\left(\Lambda_{0}\right)\right)$ è denso in $\operatorname{Im}\left(\mathcal{A}_{r, s_{1}, s_{\mathrm{a}}}\right)$.

Per ogni $f \in F_{a+2 m}^{b+2 m}\left(A_{0}\right)$, indicheremo con $\chi(f)$ una particolare soluzione dell'equazione $L(\partial) u=f$ appartenente allo spazio $F_{a}^{b}(\Lambda)$, ad esempio quella costruita col procedimento indicato al n. 3 di [2].

Inoltre per ogni $\left(f,\left\{f_{i k}\right\}\right) \in \widetilde{F}$ porremo $\tilde{\psi}_{(z)}=\left(\tilde{\varphi}_{11}\left(z+m_{11}\right), \ldots, \tilde{\varphi}_{m_{2}}\left(z+m_{m 2}\right)\right)$, dove $\left({ }^{6}\right)$

$$
\varphi_{j k}=f_{j k}-\left.P_{j k}(\partial) \chi(f)\right|_{\sigma_{k}} .
$$

Dimostrazione del teorema I. - Assegniamo $u$ tale che

$$
u \in W_{s_{1}, s_{2}}^{r}(\mathcal{A}), \quad \mathcal{A} u=\left(f,\left\{f_{j k}\right\}\right) \in \mathfrak{F}
$$

Poichè $F_{a}^{b}\left(\Lambda_{0}\right)$ è denso in $W_{s_{1}, s_{2}}^{r}(\Lambda)$, è lecito costruire una suecessione $\left(u_{n}\right)_{n \in A}$ di elementi di $F_{a}^{b}\left(\Lambda_{0}\right)$ tale che

$$
u_{n} \rightarrow u \text { in } W_{s_{1}, s_{2}}^{r}(\Lambda) \quad \text { e } \quad \mathcal{t} u_{n}=\left(f_{n},\left\{f_{j k n}\right)\right\} \rightarrow\left(f,\left\{f_{j k}\right\}\right) \text { in } E_{r, s_{1}, s_{2}}(\Lambda)
$$

Ponendo

$$
\begin{gathered}
\varphi_{j k n}=f_{j k n}-\left.P_{j k}(\partial) \chi\left(f_{n}\right)\right|_{\sigma_{k}}, \\
\tilde{\Psi}_{n}(z)=\left(\tilde{\varphi}_{11 n}\left(z+m_{11}\right), \ldots, \tilde{\varphi}_{m 2 n}\left(z+m_{m 2}\right)\right),
\end{gathered}
$$

(6) Ricordiamo (cfr. il n. 7 di [1]) che per ogni funzione $\varphi$ definita su $\sigma_{t b}$ si è convenuto di porre $\varphi_{l}(\varrho)=\varphi\left(\varrho \cos \theta_{j}\right.$, $\left.\varrho \operatorname{sen} \theta_{k}\right)$ per $\hbar=1,2$. Nel seguito, per comodità, identificheremo $\varphi \operatorname{con} \varphi_{k}$. 
per la prop. 4.1 di [2] si ha

$$
u_{n}\left(x_{1}, x_{2}\right)=\frac{1}{2 \pi i} \int_{\xi-i \infty}^{\xi+i \infty} \frac{1}{g(z)} w\left(z, x_{1}, x_{2}\right) \cdot G^{*}(z) \cdot \Psi_{n}(z) d z+\chi\left(f_{n}\right)
$$

per ogni $\left(x_{1}, x_{2}\right) \in \Lambda_{0}$ e per ogni $\left.\xi \in\right] a, b[$.

Ponendo ancora

$$
v_{n}=u_{n}-\chi\left(f_{n}\right), \quad v=u-\chi(f),
$$

dal corollario 3.1 e dalla (5.1) di [2] si ricava

e d'altra parte

$$
v_{n} \rightarrow v \quad \text { in } \quad W_{s_{1} \cdot s_{2}}^{r}(\Lambda)
$$

$$
\tilde{v}_{n, \pi}(z, \theta)=\frac{1}{g(z)} w(z, \cos \theta, \operatorname{sen} \theta) \cdot G^{*}(z) \cdot \tilde{\Psi}_{n}(z) \quad \forall z \in \sigma(a, b) .
$$

Percio, tenendo presenti le (3.9) e (3.12) di [2], si deduce che per quasi tutti i punti $(\eta, \theta) \in R \times] \theta_{1}, \theta_{2}[$ si ha

$\tilde{v}_{\pi}\left(s_{1}-r+1+i \eta, \theta\right)=$

$=\frac{1}{g\left(s_{1}-r+1+i \eta\right)} \cdot w\left(s_{1}-r+1+i \eta, \cos \theta, \operatorname{sen} \theta\right) \cdot G^{*}\left(s_{1}-r+1+i \eta\right) \cdot \tilde{\Psi}\left(s_{1}-r+1+i \eta\right)$

e che $\left(1+\eta^{2}\right)^{r / 2} \tilde{v}_{\pi}\left(s_{1}-r+1+i \eta, \theta\right)$ è di quadrato sommabile in $\left.R \times\right] \theta_{1}, \theta_{2}[$. Dal teorema di Fubini segue allora ehe, per quasi tutti i punti $\theta \in\left[\theta_{1}, \theta_{2}\right],\left(1+\eta^{2}\right)^{4 / 2}$. - $\tilde{v}_{x}\left(s_{1}-r+1+i \eta, \theta\right)$, come funzione di $\eta$, è di quadrato sommabile. Tenendo presente che $g(z)$ è infinitesima di ordine uno per $z \rightarrow z_{1}$, si deduce che per quasi tutti i punti $\theta \in\left[\theta_{1}, \theta_{2}\right]$ deve risultare

$$
w\left(z_{1}, \cos \theta, \operatorname{sen} \theta\right) \cdot G^{*}\left(z_{1}\right) \cdot \tilde{\Psi}\left(z_{1}\right)=0
$$

Ciò implica, per continuità, che in corrispondenza della radice $z_{1}$ sono verificate le condizioni (4.36) della prop. 4.8 di [2], da cui si deduce quindi la tesi.

Alla dimostrazione del teorema II premettiamo alcune considerazioni ed alcuni lemmi.

Mantenendo le posizioni e le ipotesi fatte all'inizio di questo numero, supponiamo in più che $z_{1} \notin\{-2 m+2, \ldots, 0\}$. Allora dalla dimostrazione della prop. 4.8 di [2] si deduce che perchè esista una soluzione $u \in F_{a}^{\text {tb }}\left(\Lambda_{0}\right)$ dell'equazione $A u=\left(f,\left\{f_{j k}\right\}\right)$, con $\left(f,\left\{f_{j k}\right\}\right) \in \mathfrak{F}$, è necessario e sufficiente che risulti

$$
G^{*}\left(z_{1}\right) \cdot \tilde{\Psi}\left(z_{1}\right)=0
$$


LEMMA 1.1. - Se z $z_{1}$ è radice semplice della funzione discriminante g, la matrice $G\left(z_{1}\right)$ ha caratteristica uguale a $2 m-1$.

Infatti, teniamo presente che ogni elemento della matrice $G(z)$ è una funzione olo ${ }^{2}$ morfa in tutto il piano complesso. Posto

$$
g_{i j}^{k h}(z)=\sum_{r=0}^{\infty}\left(g_{i j}^{k h}\right)^{(r)}\left(z_{1}\right) \frac{\left(z-z_{1}\right)^{r}}{r !}
$$

si riconosce con ovvie considerazioni che, se la caratteristica della matrice $G\left(z_{1}\right)$ fosse inferiore a $2 m-1$, il determinante della matrice $G(z)$ sarebbe divisibile per $\left(z-z_{1}\right)$ almeno. Ciò prova l'asserto.

LeITMA 1.2. - Se $z_{1}$ è radice semptice della funzione discriminante $g$, esistono $2 m$ costanti $c_{11}, \ldots, c_{m 2}$, non tutte nulle, tali che le $2 m$ condizioni scalari cui si riconduce la (1.4) sono verificate se e soltanto se

$$
\sum_{k=1}^{2} \sum_{j=1}^{m} c_{j k} \int_{0}^{+\infty} \varphi_{j k}(t) t^{z_{1}+m_{j k}-1} d t=0
$$

Indichiamo $\operatorname{con} g_{i j}^{* h k}(z)$ il complemento algebrico di $g_{j i}^{k h}(z)$ nella matrice $G(z)$. Dal lemma 1.1 segue che esistono $i \in\{1, \ldots, m\}$ e $h \in\{1,2\}$ per cui non tutti i numeri $g_{i 1}^{*_{11}}\left(z_{1}\right), \ldots, g_{i m}^{*_{2}}\left(z_{1}\right)$ sono nulli. Se ne deduce che

$$
\sum_{h=1}^{m} \sum_{j=1}^{2} g_{i j}^{* h z}\left(z_{1}\right) \tilde{\Psi}_{j k}\left(z_{1}\right)=0
$$

esprime la condizione necessaria e sufficiente perchè il sistema di equazioni lineari

$$
\sum_{h=1}^{2} \sum_{l=1}^{m} g_{j l}^{k h}\left(z_{1}\right) \gamma_{l h}=\tilde{\Psi}_{j k}\left(z_{1}\right), \quad j=1, \ldots, m, k=1,2
$$

nelle incognite $\gamma_{11}, \ldots, \gamma_{m 2}$ abbia soluzione.

D'altra parte il sistema (1.7) è compatibile se e solo se è verificata la (1.4), e perciò la (1.4) è equivalente alla (1.6). Esplicitando quest'ultima attraverso le trasformate di Mellin $\tilde{\phi}_{j k}\left(z+m_{j k}\right)$ si trova la $(1.5)$ con $c_{j k}=g_{i j}^{* h k}\left(z_{1}\right)$ per $j=1, \ldots, m$ e $k=1,2$.

OsServazione 1.1. - Rileviamo che mentre il funzionale definito in $\mathfrak{F}$ dal primo membro della (1.5) e dalla (1.3) si prolunga per continuità a tutto $E_{r, s_{1}^{\circ}, s_{2}}(\Lambda)$, per il fatto che $s_{1}^{0}-r+1<\operatorname{Re} z_{1}<s_{2}-r+1$, conformemente a quanto abbiamo stabilito con i lemmi 6.1 e 6.2 di [2], tale funzionale non ha alcun prolungamento continuo a $E_{r, s_{1}, s_{1}}(\Lambda)$. Ciò si riconosce facilmente osservando che se $\left(\varphi_{j k, n}\right)_{n \in \mathbb{N}}$ converge a $\varphi_{j k}$ 
in $W_{s_{1}, s_{2}}^{r-m j_{k}-\frac{1}{2}}\left(\sigma_{k}\right)$, la successione di integrali

$$
\int_{0}^{+\infty} \varphi_{j k, n}(t) t^{s_{1}-r+m_{j k}+i \eta} d t
$$

può addirittura non convergere, per il fatto che $\varphi_{g_{k}}(t) t^{s_{1}-r+m_{9 k}+i \eta}$ può non essere sommabile in un intorno destro dell'origine.

Lemma 1.3. - Siano: $B_{1}$ e $B_{2}$ due spazi di Banach, $B_{1}$ immerso in $B_{2}$ algebricamente e topologicamente, $B_{1}$ denso in $B_{2} ; w_{1}, \ldots, w_{m} \in B_{1}^{\prime}$ (= duale forte di $B_{1}$ ) linearmente indipendenti e tali ohe $w_{j}$, per $j=1, \ldots, m$, non abbia prolungamento continuo a $B_{2}$. Posto

$$
N_{m}=\left\{x \in B_{1} \mid\left\langle x, w_{i}\right\rangle=0 \text { per } j=1, \ldots, m\right\},
$$

si ha ehe $N_{m}$ è denso in $B_{2}$.

Si ragiona per induzione. Fissato $N_{1}$, si consideri $x_{1} \in B_{1}$ tale che $\left\langle x_{1}, w_{1}\right\rangle=1$. Si ha allora

$$
B_{1}=N_{1} \oplus M_{1} \quad \text { con } \quad M_{1}=\left\{e x_{1}\right\}_{c \in \mathbb{I}},
$$

essendo $K$ il campo degli scalari relativo agli spazi vettoriali $B_{1}$ e $B_{2}$. Proviamo che $N_{1}$ è denso in $B_{2}$. Ragioniamo per assurdo. Se $N_{1}$ non fosse denso in $B_{2}$ la chiusura $\bar{N}_{1}$ di $N_{1}$ in $B_{2}$ sarebbe un sottospazio proprio di $B_{2}$. Sia allora $\Phi$ un funzionale lineare, continuo e non nullo su $B_{2}$ tale che

$$
\langle x, \Phi\rangle=0 \quad \forall x \in \bar{N}_{1} .
$$

Posto per ogni $x \in B_{1}$

$$
x=x^{\prime}+\left\langle x, w_{1}\right\rangle x_{1},
$$

si ha $x^{\prime} \in N_{1}$ e conseguentemente $\left\langle x^{\prime}, \Phi\right\rangle=0$. Perciò

$$
\langle x, \Phi\rangle=\left\langle x, w_{1}\right\rangle \cdot\left\langle x_{1}, \Phi\right\rangle \quad \forall x \in B_{1}
$$

Non può essere $\left\langle x_{1}, \Phi\right\rangle=0$ perchè altrimenti $\Phi$ sarebbe identicamente nullo su $B_{1}$, ed essendo $\Phi$ continuo in $B_{2}$ e $B_{1}$ denso in $B_{2}$ risulterebbe $\Phi$ identicamente nullo su $B_{2}$, contrariamente alla condizione imposta a $\Phi$. Se ne deduce allora che $w_{1}=\left(\left\langle x_{1}, \Phi\right\rangle\right)^{-1} \Phi$ su $B_{1}$. Ciò però è in contrasto con l'ipotesi, perchè implica che $w_{1}$ si può prolungare per continuità a tutto $B_{2}$. L'assurdo cui siamo pervenuti prova l'asserto nel caso $m=1$.

Supponiamo d'aver dimostrato il teorema per $m=k$. Introduciamo in $B_{1} k+1$ elementi $x_{1}, \ldots, x_{k+1}$ in modo che

$$
\left\langle x_{i}, w_{l}\right\rangle=\delta_{i l} \quad \text { per } \quad j, l=1, \ldots, k+1 .
$$


Considerato il sottospazio vettoriale di $B_{1}$

$$
M_{k}=\left\{c_{1} x_{1}+\ldots+c_{k} x_{k}\right\}_{\left(c_{1} \ldots, c_{k}\right) \in \mathbb{R}^{k}},
$$

risulta

$$
B_{1}=N_{k} \oplus M_{k}
$$

e, per il ragionamento di induzione che stiamo facendo, $N_{k}$ è denso in $B_{2}$ : Per costruzione risulta $x_{k+1} \in N_{k}$ e perciò anche $M_{k+1}^{\prime}=\left\{c x_{k+1}\right\}_{c \in \boldsymbol{K}}$ è un sottospazio di $N_{k}$ e risulta pertanto

$$
N_{k}=N_{k+1} \oplus M_{k+1}^{\prime} .
$$

Se $N_{k+1}$ non fosse denso in $B_{2}$, esisterebbe un funzionale $\Phi$ lineare, continuo e non nullo su $B_{2}$ tale che $\langle x, \Phi\rangle=0$ per ogni $x \in \bar{N}_{k+1}$.

Allora posto

$$
x=x^{\prime}+\left\langle x, w_{k+1}\right\rangle x_{k+1} \quad \forall x \in N_{k},
$$

si avrebbe

$$
\langle x, \Phi\rangle=\left\langle x, w_{k+1}\right\rangle \cdot\left\langle x_{k+1}, \Phi\right\rangle \quad \forall x \in N_{k} .
$$

Se fosse $\left\langle o_{k+1}, \Phi\right\rangle=0, \Phi$ sarebbe identicamente nullo su $N_{k}$ e, poichè $N_{k}$ è denso in $B_{2}$, sarebbe $\Phi$ identicamente nullo su $B_{2}$, contrariamente alla costruzione adottata per $\Phi$. Percio $\left\langle x_{k+1}, \Phi\right\rangle \neq 0$. Se ne deduce

$$
\left\langle x, w_{k+1}\right\rangle=\left(\left\langle x_{k+1}, \Phi\right\rangle\right)^{-1}\langle x, \Phi\rangle \quad \forall x \in N_{k} .
$$

Essendo $\Phi$ continuo in $B_{2}$, ciò implica che $w_{k+1}$ può essere prolungato in un funzionale continuo su $B_{2}$, contrariamente all'ipotesi fatta. L'assurdo cui siamo pervenuti prova che $N_{k+1}$ è denso in $B_{2}$ e perciò si ha la tesi.

Dimostrazione del teorema II. - Consideriamo il funzionale $\Phi$ definito in $E_{r, s_{i}^{\prime}, s_{2}}(\Lambda)$ dal primo membro della (1.5), cioè

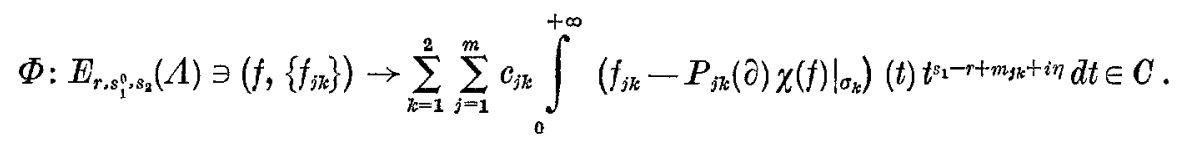

Per quanto abbiamo sopra osservato (cfr. l'osservazione 1.1) $\Phi$ non ha prolungamento continuo ad $E_{r, s_{1}, s_{2}}(\Lambda)$, perciò, per il lemma 1.3 , il sottospazio

$$
\mathcal{N}_{\Phi}=\left\{\left(f,\left\{f_{i k}\right\}\right) \in E_{r, s_{1}^{o} \cdot s_{2}}(\Lambda) \mid \Phi\left(f,\left\{f_{i k}\right\}\right)=0\right\}
$$

è denso in $E_{r, s_{1}, s_{2}}(\Lambda)$. 
D'altra parte dal lemma 1.2 e da quanto si è detto a proposito della (1.4) si deduce $\mathcal{A}\left(F_{a}^{b}\left(\Lambda_{0}\right)\right) \subset \mathcal{N}_{\Phi}$ e, per le costruzioni fatte ed il teorema 1.1. di [2], si ha anche $\mathcal{N}_{\Phi}=\operatorname{Im}\left(\mathcal{A}_{r, s_{1}, s_{2}}\right)$ e quindi risulta

$$
\mathcal{A}\left(F_{a}^{b}\left(\Lambda_{0}\right)\right) \subset \mathcal{N}_{\Phi}=\operatorname{Im}\left(\mathcal{A}_{r, s_{1}^{0}, s_{s}}\right) \subset \operatorname{Im}\left(\mathcal{A}_{r, s_{1}, s_{2}}\right)
$$

Poichè $F_{a}^{\grave{b}}\left(\Lambda_{0}\right)$ è denso in $W_{s_{1}, s_{\mathrm{a}}}^{r}(\Lambda)$, la chiusura di $\mathcal{A}\left(F_{a}^{b}\left(\Lambda_{0}\right)\right)$ in $E_{r, s_{1}, s_{2}}(\Lambda)$ contiene $\operatorname{Im}\left(\mathcal{A}_{r, s_{1}, s_{2}}\right)$. Se, per assurdo, supponiamo che $\operatorname{Im}\left(\mathcal{A}_{r, s_{1}, s_{2}}\right)$ è chiusa in $E_{r, s_{1}, s_{2}}(\Lambda)$, per il fatto che $\mathcal{N}_{\Phi}$ è denso in $E_{r, s_{1}, s_{2}}(\Lambda)$ si dovrà avere da (1.8)

$$
\overline{\mathcal{A}\left(F_{a}^{b}\left(\Lambda_{0}\right)\right)}=\operatorname{Im}\left(\mathcal{A}_{r, s_{1}, s_{2}}\right)=E_{r, s_{1}, s_{2}}(\Lambda)
$$

D'altra parte esiste certamente $\left(f,\left\{f_{j l}\right\}\right) \in \mathfrak{F}$ tale che

$$
\Phi\left(f,\left\{f_{j k}\right\}\right) \neq 0 \text {. }
$$

Poichè vale la (1.2), dalla (1.9) si deduce anche $\left(f,\left\{f_{i k}\right\}\right) \in \operatorname{Im}\left(\mathcal{A}_{r, s_{1}, s_{2}}\right)$ e perciò

$$
\exists u \in W_{s_{1}, s_{\mathrm{a}}}^{r}(\Lambda) \ni^{\prime} A u=\left(f,\left\{f_{j k}\right\}\right)
$$

Ma allora dal teorema I segue $u \in H_{a}^{b}\left(\Lambda_{0}\right)$ e quindi $\Phi\left(f,\left\{f_{j k}\right\}\right)=0$, in contrasto con la (1.10); ciò prova l'asserto.

\section{2. - Dimostrazione del teorema IV.}

Premettiamo alcune considerazioni ed alcuni lemmi.

Ricordiamo (efr. il teorema 1.1, di [2]) ehe le condizioni di compatibilità per l'equazione $\mathfrak{A}_{r, s_{1}, s_{\mathrm{s}}} u=\left(f,\left\{f_{j k}\right\}\right)$ sono determinate dalle radici $z_{j}$ della funzione discriminante $g$ associata a $\left(L,\left\{P_{j k}\right\}\right)$, che appartengono alla striscia $\sigma\left(s_{1}-r+1, s_{2}-r+1\right)$. Scriveremo ora tali condizioni sotto la forma

$$
\left(F_{j l} \circ \Psi\right)\left(f,\left\{f_{j k}\right\}\right)=0, \quad l=1, \ldots, n_{j}, j=1, \ldots, v
$$

conformemente alle notazioni che abbiamo introdotto a proposito del lemma 6.2 di [2]. Perciò $v$ rappresenta il numero delle radici distinte che $g$ ha in $\sigma\left(s_{1}-r+1, s_{2}-r+1\right)$ e $n_{j}$ il massimo numero di condizioni portate dalla radice $z_{j}$.

LeMma 2.1. - Se $z_{q}$ e $z_{j}$ sono due radici distinte di g appartenenti a $\sigma\left(s_{1}-r+1\right.$, $\left.s_{2}-r+1\right)$, ogni funzionale $F_{q l} \circ \Psi$ è linearmente indipendente dall'insieme di funzionali $F_{j 1} \circ \Psi, \ldots, F_{j, n_{j}} \circ \Psi$.

$$
11 \text { - Annali di Matematica }
$$


Tenendo presente la forma che assumono i funzionali $F_{j l}$ (cfr. 1.c.), indichiamo con $p_{i l}$ dei polinomi generici; si riconosce facilmente che, per provare il lemma, basta dimostrare che ogni funzione del tipo $t^{z_{a}} p_{q, l}(\log t)$, per $t \in R_{+}$e $p_{q l}$ polinomio non nullo, è linearmente indipendente da un insieme di funzioni del tipo $t^{z_{j}} p_{j 1}(\log t), \ldots$, $t^{z} p_{j n}(\log t)$. Tale affermazione si stabilisce facilmente sfruttando la condizione $z_{j} \neq z_{q}$.

Indichiamo ora con $L_{\varepsilon}(\partial)$ l'operatore differenziale definito dalla (5.28) di [2]; ricordiamo che $L_{\varepsilon}(\zeta, 1)$ ha tutte le radici semplici, per $\varepsilon$ positivo ma abbastanza piccolo, denotate con $\beta_{j, k, \varepsilon}$, definite dalla (5.27) di [2], continue per $\varepsilon \rightarrow 0^{+}$e tali che $\beta_{j, k, 0}=\beta_{j k}$. Sia inoltre $g_{\varepsilon}$ la funzione discriminante associata a $\left(L_{\varepsilon},\left\{P_{j k}\right\}\right)$ ed $\mathcal{A}_{\gamma, s_{1}, s_{2}}^{\varepsilon}$ l'applicazion" cosi definita:

$$
\mathcal{A}_{r, s_{1}, s_{2}}^{\varepsilon}: W_{s_{1}, s_{3}}^{r}(\Lambda) \ni u \rightarrow\left(L_{\varepsilon}(\partial) u, P_{11}(\partial) u\left|\sigma_{1}, \ldots, P_{m 2}(\partial) u\right|_{\sigma_{2}}\right) \in E_{r, s_{1}, s_{2}}(\Lambda)
$$

LEMMA 2.2. - Se $s_{1}<s_{2}$ e $s_{i}-r+1 \notin \Sigma_{g}$ per $i=1,2$, è possibile fissare $\varepsilon_{0} \in R_{+}$ in modo che per ogni $\varepsilon \in\left[0, \varepsilon_{0}\right]$ risulti:

a) $\mathcal{A}_{r, s_{1}, s_{2}}^{\varepsilon}$ è un operatore ad indice;

b) $g_{s}(z)$ e $g(z)$ hanno in $\sigma\left(s_{1}-r+1, s_{2}-r+1\right)$ lo stesso numero di zeri $\left(^{7}\right)$;

c) indice $\left(\mathcal{A}_{r, s_{1}, s_{2}}^{\varepsilon}\right)=$ indice $\left(\mathcal{A}_{r, s_{1}, s_{2}}\right)$.

Osserviamo che $L_{\varepsilon}(\partial)$ ha i coefficienti che sono funzioni continue di $\varepsilon$ e che $L_{0}(\partial)$ coincide con $L(\partial)$. Le proposizioni 4.6 e 4.7 ed il teorema 1.1 di [2] consentono di provare direttamente che esiste $\varepsilon_{0}^{\prime} \in R_{+}$in modo che per ogni $\varepsilon \in\left[0, \varepsilon_{0}^{\prime}\right]$ sono vere a) $\mathrm{e} b$ ).

Poichè la citata continuità dei coefficienti di $L_{\varepsilon}(\partial)$ implica che $\mathfrak{A}_{r, s_{1}, s_{2}}^{\varepsilon}$ converge in norma ad $\mathcal{A}_{r, s_{1}, s_{2}}$ per $\varepsilon \rightarrow 0^{+}$, per il teorema 2.2 di [4] è possibile fissare un numero reale positivo $\varepsilon_{0} \leqslant \varepsilon_{0}^{\prime}$ in modo che sussista la $c$ ) per ogni $\varepsilon \in\left[0, \varepsilon_{0}\right]$. Ciò prova completamente l'asserto.

Supponiamo ora che le radici del polinomio $L(\zeta, 1)$ siano tutte semplici. Mettiamo in evidenza la dipendenza della funzione discriminante $g$ dalle radici di $L(\zeta, 1)$ scrivendo anche

$$
g(z)=g\left(z ; \beta_{11}, \ldots, \beta_{m 2}\right) .
$$

Poichè $g$ dipende analiticamente dalle radici, almeno finchè queste conservano parte immaginaria diversa da zero, ha significato considerare la funzione

$$
\gamma(z, \lambda)=g\left(z ; \lambda, \beta_{21}, \ldots, \beta_{m 2}\right), \quad \text { per } \quad z \in O \text { e } \operatorname{Im} \lambda>0
$$

Si può interpretare $\gamma(z, \lambda)$ come funzione diseriminante associata a $\left(L^{\lambda},\left\{P_{j k}\right\}\right)$, ove $L^{\lambda}(\zeta, 1)$ denota il polinomio che si ottiene da $L(\zeta, 1)$ sostituendo la radice $\beta_{11}$ con $\lambda$, ossia $L^{\lambda}(\zeta, 1)=(\zeta-\lambda) L(\zeta, 1)\left(\zeta-\beta_{11}\right)^{-1}$.

(") Si intende che ogni zero è contato tante volte quanto il suo ordine di molteplicità. 
Evidentemente i coefficienti di $L^{\lambda}(\zeta, 1)$ sono funzioni continue di $\lambda$ e $L^{\beta_{11}}(\zeta, 1)=$ $=L(\zeta, 1)$. Fissati $\alpha \in C$ e $\delta \in R_{+}$, denotiamo con $O_{\alpha, \delta}$ il cerchio del piano complesso col centro in $\alpha$ e raggio $\delta$, ossia $C_{\alpha, \delta}=\{z \in C|| z-\alpha \mid \leqslant \delta\}$. Fissiamo $\delta_{0} \in R_{+}$in modo che $C_{\beta_{11}, \delta_{0}}$ sia contenuto nel semipiano $\operatorname{Im} z>0$ e non ci siano in esso altri zeri di $L(\zeta, 1)$ fuorchè $\beta_{11}$. Per ogni $\lambda_{1} \in C_{\beta_{11}, \delta_{0}}$ poniamo

$$
g_{\varepsilon}^{\lambda_{1}}(z)=\gamma\left(z ; \beta_{11}+\varepsilon\left(\lambda_{1}-\beta_{11}\right)\right), \quad L_{\varepsilon}^{\lambda_{1}}=L^{\beta_{11}+\varepsilon\left(\lambda_{1}-\beta_{11}\right)}, \quad \varepsilon \in[0,1]
$$

Evidentemente i coefficienti di $L_{\varepsilon}^{\lambda_{1}}$ sono funzioni continue di $\varepsilon, L_{0}^{\lambda_{1}}=L$ e $g_{\varepsilon}^{\lambda_{1}}(z)$ è la funzione discriminante associata a $\left(I_{\varepsilon}^{\lambda_{1}},\left\{P_{j k}\right\}\right)$.

LEMMA 2.3. - Se $s_{1}<s_{2}$ e $s_{i}-r+1 \notin \Sigma_{g}$ per $i=1,2$, è possibile fissare $\lambda_{1} \in C_{\beta_{11}, \delta_{a}}$ ed $\varepsilon_{0} \in R_{+}$in modo che per ogni $\left.\left.\varepsilon \in\right] 0, \varepsilon_{0}\right]$ risulti

$\left.a_{1}\right) g_{\varepsilon}^{\lambda_{1}}(z)$ e $g(z)$ hanno in $\sigma\left(s_{1}-r+1, s_{2}-r+1\right)$ lo stesso numero di zeri e $g_{\varepsilon}^{\lambda_{1}}(z)$, come $g(z)$, non si annulla sui lati di $\sigma\left(s_{1}-r+1, s_{2}-r+1\right)$.

$\left.a_{2}\right)$ Gli zeri che $g_{s}^{\lambda_{1}}(z)$ ha in $\sigma\left(s_{1}-r+1, s_{2}-r+1\right)$ sono tutti semplici.

Indichiamo con $z_{1}, \ldots, z_{y}$ gli zeri distinti che $g(z)$ ha nella striseia $\sigma\left(s_{1}-r+1\right.$, $s_{2}-r+1$ ) e denotiamo con $p_{i}$ l'ordine di molteplicità di $z_{j}$. Fissiamo un numero reale positivo $\varrho_{0}$ in modo che i cerchi $C_{z_{j}, \varrho_{0}}$ siano a due a due disgiunti e tutti contenuti in $\sigma\left(s_{1}-r+1, s_{2}-r+1\right)$. Osserviamo ora che, per la funzione olomorfa $\gamma(z, \lambda)$ delle due variabili complesse $z$ in $C$ e $\lambda$ nel semipiano $\operatorname{Im} \lambda>0$, è verificata la proprietà: $\gamma\left(z, \beta_{11}\right)$ ha nel punto $z=z_{j}$ uno zero di ordine di molteplicità $p_{j}$ per ogni $j \in\{1, \ldots, v\}$. Per un noto teorema di Weierstrass, per ogni $j \in\{1, \ldots, v\}$, è possibile fissare due numeri reali positivi $\varrho_{j}\left(\leqslant \varrho_{0}\right), \delta_{j}\left(\leqslant \delta_{0}\right)$ ed un polinomio

$$
F_{j}(z, \lambda)=z^{p_{j}}+\alpha_{j 1}(\lambda) z^{p_{j}-1}+\ldots+\alpha_{i p_{j}}(\lambda)
$$

in modo che, comunque si fissi $\lambda^{\prime} \in C_{\beta_{1_{1}, \delta_{j}}}$, il polinomio in $z F_{j}^{\prime}\left(z, \lambda^{\prime}\right)$ ha $p_{j}$ zeri appartenenti al cerchio $C_{z_{j}, e_{j}}$, i quali sono gli unici zeri che la funzione in $z \gamma\left(z, \lambda^{\prime}\right)$ ha nel cerchio $C_{z_{j}, \varrho_{j}}$; inoltre le funzioni $\alpha_{j 1}(\lambda), \ldots, \alpha_{j p_{j}}(\lambda)$ sono olomorfe in $C_{\beta_{11}, \delta_{j}}$.

Sia allora $A_{j}(\lambda)$ il risultante associato a $F_{j}(z, \lambda)$ e $\partial_{z} F_{j}(z, \lambda)$, come polinomi in $z$. Poichè $\Delta_{j}(\lambda)$ è una funzione olomorfa in $C_{\beta_{11}, \delta_{j}}$ e non identicamente nulla, è possibile fissare un numero reale positivo $\delta \leqslant \min \left\{\delta_{1}, \ldots, \delta_{v}\right\}$ in modo che ciascuna funzione $\Delta_{j}(\lambda)$ non abbia alcuno zero in $C_{\beta_{11}, \delta}$.

Ciò comporta che, per ogni $\lambda \in O_{\beta_{1}, \delta}, F_{j}(z, \lambda)$ ha i $p_{j}$ zeri, già appartenenti a $O_{z j, 0_{j}}$, a due a due distinti.

Fissiamo $\lambda_{1} \in C_{\beta_{11}, \delta}$ ed osserviamo che per ogni $\varepsilon \in[0,1]$ si ha $\beta_{11}+\varepsilon\left(\lambda_{1}-\beta_{11}\right) \in C_{\beta_{11}, \delta}$. Se ne deduce che la funzione $g_{\varepsilon}^{\lambda_{1}}(z)$ per ogni $\varepsilon \in[0,1]$ ha nella striscia $\sigma\left(s_{1}-r+1\right.$, $\left.s_{2}-r+1\right) p_{1}$ zeri semplici in $\sigma_{z_{1}, \varrho_{1}}, p_{2}$ zeri semplici in $O_{z_{3}, \ell_{2}}, \ldots, p_{v}$ zeri semplici in $C_{z, e_{v}}$. La tesi si deduce allora facilmente dalle proposizioni 4.6 e 4.7 di [2]. 
Dimostrazione del teorema IV. - Intanto, in conseguenza del lemma 2.2, per provare l'asserto basta mettersi nel caso in cui tutte le radici del polinomio $L(\zeta, 1)$ siano semplici. D'altra parte il lemma 1.2 ed il lemma 2.1 provano che l'asserto è vero se la funzione discriminante ha nella striscia $\sigma\left(s_{1}-r+1, s_{2}-r+1\right)$ soltanto zeri semplici. Consideriamo allora l'applicazione $\mathcal{A}_{r, s_{1}, s_{2}}^{\lambda_{1}, \varepsilon} \operatorname{così~definita:~}$

$$
\mathcal{A}_{r_{3}, s_{1}, \varepsilon_{3}}^{\lambda_{1}, \varepsilon}: W_{s_{1}, s_{2}}^{r}(\Lambda) \ni u \rightarrow\left(L_{\varepsilon}^{\lambda_{1}}(\partial) u,\left.P_{11}(\partial) u_{1}\right|_{\sigma_{1}}, \ldots,\left.P_{m_{2}}(\partial) u\right|_{\sigma_{2}}\right) \in E_{r, s_{1}, s_{2}}(\Lambda),
$$

essendo $L_{\varepsilon}^{\lambda_{1}}(\partial)$ l'operatore sopra definito (cfr. la (2.3)). Si riconosce facilmente che $\mathcal{A}_{r, s_{2}, s_{2}}^{\lambda_{1}, \varepsilon}$ per $\varepsilon \rightarrow 0^{+}$converge in norma a $\mathfrak{A}_{r, s_{1}, s_{2}}$. Perciò, ancora per il teorema 2.2 di [4], è possibile determinare un $\left.\left.\varepsilon_{1} \in\right] 0, \varepsilon_{0}\right]$ tale che $\mathscr{A}_{r, s_{1}, s_{2}}^{\lambda_{\lambda_{2}, \varepsilon_{1}}}$ sia un operatore ad indice e indice $\left(\mathcal{A}_{r, s_{1}, s_{\mathrm{a}}}^{\lambda_{1}, \varepsilon_{1}}\right)=$ indice $\left(\mathcal{A}_{r, s_{1}, s_{s}}\right)$, dove $\varepsilon_{0}$ è il numero definito nel lemma 2.3. Da quest'ultimo lemma e da quanto è stato già osservato a proposito del caso in cui le radici della funzione discriminante sono semplici, segue evidentemente la tesi.

La formula dell'indicatore logaritmico consente di dare una espressione analitica per l'indice dell'operatore $\mathfrak{A}_{r, s_{1}, s_{2}}$ nel caso in cui $s_{i}-r+1 \notin \Sigma_{g}(i=1,2)$. Posto infatti $I_{r, s_{1}, s_{2}}\left(\eta_{0}\right)=\left[s_{1}-r+1, s_{2}-r+1\right] \times\left[-\eta_{0}, \eta_{0}\right]$, consideriamo $\eta_{0} \in R_{+}$in modo ehe tutti gli zeri che $g$ ha in $\sigma\left(s_{1}-r+1, s_{2}-r+1\right)$ siano interni a $I_{r, s_{1}, s_{2}}\left(\eta_{0}\right)$. Dal teorema IV si deduce allora

$$
\text { indice }\left(\mathfrak{t}_{r, s_{1}, s_{2}}\right)=-\frac{1}{2 \pi i} \int_{+\partial I_{r_{s}, s_{1}, s_{2}\left(\eta_{0}\right)}} \frac{g^{\prime}(z)}{g(z)} d z=-\lim _{\eta_{0} \rightarrow+\infty} \frac{1}{2 \pi i} \int_{+\partial I_{r}, s_{1}, s_{2}\left(\eta_{0}\right)} \frac{g^{\prime}(z)}{g(z)} d z \text {. }
$$

\section{3. - Dimostrazione del teorema III.}

Riprendendo alcune notazioni e posizioni del n. 2 di [3], consideriamo un ricoprimento di $\bar{\Lambda}$ mediante un numero finito di sottoinsiemi aperti $\mathcal{O}_{1}, \ldots, \mathcal{O}_{v}$ di $R^{2}$, con la condizione che $\mathcal{O}_{1}, \mathcal{O}_{3}, \ldots, \mathcal{O}_{y}$ siano limitati e l'origine appartenga soltanto a $\mathcal{O}_{1}$, e assegniamo una partizione dell'unità in $C^{\infty}(\bar{A})$, subordinata a tale ricoprimento, mediante le funzioni $\zeta_{1}, \ldots, \zeta_{p}$ tali che

$$
\begin{aligned}
& \zeta_{i} \in C^{\infty}\left(R^{2}\right), \quad \operatorname{supp} \zeta_{i} \subset \mathcal{O}_{i}, \\
& \zeta_{i}(x) \geqslant 0, \quad \sum_{i=1}^{\nu} \zeta_{i}(x)=1 \quad \forall x \in \bar{A} .
\end{aligned}
$$

Inoltre fissiamo dei punti $x^{1}, \ldots, x^{y}$, con $x^{1}=0, x^{2}=\infty$ e $x^{i} \in \mathcal{O}_{i} \cap A$ per $i=3, \ldots, v$, e poniamo

$$
A_{i}^{\prime}=A^{\prime}\left(x^{i}, \partial\right), \quad B_{i j k}^{\prime}=B_{j k}^{\prime}\left(x^{i}, \partial\right), \quad i=1, \ldots, \nu,
$$

dove $A^{\prime}(x, \partial)$ e $B_{j k}^{\prime}(x, \partial)$ denotano le parti principali di $A(x, \partial)$ e $B_{j k}(x, \partial)$ rispettivamente. 
Poniamo ancora, per ogni $i \in\{1, \ldots, \nu\}$,

$$
\Psi_{i}(v)=\left\|\left(A^{\prime}-A_{i}^{\prime}\right) v\right\|_{F_{s_{1}, s_{2}}^{r-2 m}(A)}+\sum_{j=1}^{m} \sum_{k=1}^{2}\left\|\left(B_{j k}^{\prime}-B_{i j k}^{\prime}\right) v\right\|_{W_{s_{1}, s_{2}}^{r-m} m_{j k}-\frac{1}{2}\left(\sigma_{k}\right)} .
$$

Per la (2.2) di [3] si ha che, fissato un numero reale $\eta>0$, è possibile scegliere $\nu$ e gli aperti $\mathcal{O}_{1}, \ldots, \mathcal{O}_{v}$ in modo che si abbia

$$
\Psi_{i}\left(\zeta_{i} u\right) \leqslant \eta\left\|\zeta_{i} u\right\|_{W_{s_{1}, s_{s}(A)}^{r}} \quad \forall u \in W_{s_{1}, s_{s}}^{r}(\Lambda), \quad i=1, \ldots, v
$$

Passando ora alla dimostrazione del teorema III, supponiamo per assurdo che, per un $i \in\{1,2\}, s_{i}-r+1$ appartenga a $\Sigma_{i}$ e non sia uno zero reale di $g_{i}$ appartenente a $\{-2 m+2, \ldots, 0\}$ e che la (1.14) di [3] sia vera con $\varepsilon>0$.

Assegnata allora $u \in \mathfrak{D}(\bar{\Lambda} \backslash\{0\})$ e posto

$$
\mathcal{A} u=\left(A u,\left\{\left.B_{j k} u\right|_{\sigma_{k}}\right\}\right), \quad \mathcal{A}^{i} u=\left(A_{i}^{\prime} u,\left\{\left.B_{i j k}^{\prime} u\right|_{\sigma_{k}}\right\}\right) \quad \text { e } \quad u_{i}=\zeta_{i} u,
$$

si ha per ogni $i \in\{1, \ldots, \nu\}\left(^{8}\right)$

$$
\begin{aligned}
& \left\|u_{i}\right\|_{\Psi_{s_{1}, s_{i}}^{r}(A)} \leqslant c_{1}\left(\left\|\mathcal{A} u_{i}\right\|_{g_{r_{,}, s_{1}, s_{2}}(A)}+\left\|u_{i}\right\|_{L_{\left(s_{1}+s_{2}\right) / 2-r, \cdots\left(s_{2}-s_{1}\right) / 2+\varepsilon}^{2}(A)}\right) \leqslant \\
& \leqslant c_{1}\left(\left\|\mathcal{A}^{i} u_{i}\right\|_{s_{r, s_{1}, s_{2}}(A)}+\Psi_{i}\left(u_{i}\right)+\left\|A^{\prime \prime} u_{i}\right\|_{W_{s_{1}, s_{1}}^{r-2 m}(A)}+\sum_{j=1}^{m} \sum_{k=1}^{2}\left\|B_{j k}^{\prime \prime}\right\|_{W_{s_{1}, s_{2}}^{r-m_{j}-\frac{1}{2}\left(\sigma_{k}\right)}}+\right. \\
& \left.+\left\|u_{i}\right\|_{L_{\left(s_{1}+s_{2}\right) / 2-r_{1}-\left(s_{2}-s_{1}\right) / 2+\varepsilon}^{2}(\Lambda)}\right),
\end{aligned}
$$

dove si è posto

$$
A^{\prime \prime}=A-A^{\prime}, \quad B_{j k}^{\prime \prime}=B_{j k}-B_{j k}^{\prime} .
$$

Da tale relazione, dalle (2.7)-(2.13) di [3] e dalla (3.1) scritte per $\eta$ sufficientemente piccolo si deduce la limitazione

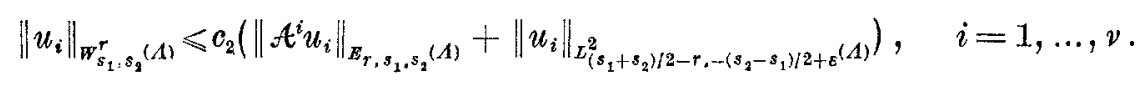

Mostriamo inoltre che, fissato $t_{2} \geqslant s_{1}$ e tale che $t_{2}-r+1 \notin \Sigma_{1}$, si ha per ogni $i \in\{1, \ldots, v\}$

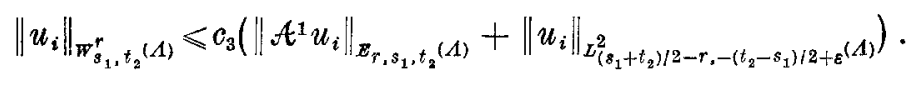

$\left.{ }^{8}\right)$ Nel corso di questa dimostrazione denoteremo con $c_{1}, \ldots, c_{6}$ delle costanti indipendenti da $u$. 
Infatti, tale relazione per $i=1$ segue dalla (3.3) e daI teorema 8.4 di [1], mentre per $i=2, \ldots, v$ segue dal teorema 1.1 di [3] e ancora dal teorema 8.4 di [1].

In modo analogo si prova che, fissato $t_{1} \leqslant s_{2}$ e tale che $t_{1}-r+1 \notin \Sigma_{2}$, si ha per ogni $i \in\{1, \ldots, v\}$

$$
\left\|u_{i}\right\|_{T_{t_{1}, s_{2}}^{r}(A)} \leqslant c_{\mathcal{A}}\left(\left\|\mathcal{A}^{2} u_{i}\right\|_{E_{r, t_{1}, s_{2}}(A)}+\left\|u_{i}\right\|_{L_{\left(t_{1}+s_{3}\right) / 2-r,-\left(s_{2}-t_{1}\right) / 2+\varepsilon^{2}(A)}^{2}}\right)
$$

Con ragionamenti analoghi a quelli tenuti in [3] per dedurre dalla (2.14) la (1.14), si deduce ora dalla (3.4) la limitazione

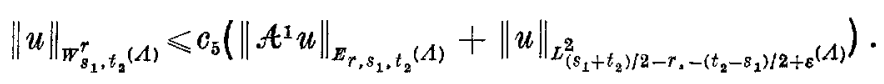

per ogni $u \in \mathscr{D}(\bar{\Lambda} \backslash\{0\})$ e quindi anche per ogni $u \in W_{s_{1}, t_{2}}^{r}(\Lambda)$.

Analogamente dalla (3.5) si deduce la limitazione

$$
\|u\|_{W_{t_{1}, s_{2}}^{r}(\Lambda)} \leqslant c_{6}\left(\left\|\mathcal{A}^{2} u\right\|_{E_{r, t_{1}, s_{2}}(\Lambda)}+\|u\|_{L_{\left(t_{1}+\varepsilon_{2}\right) / 2-r_{2}-\left(s_{2}-t_{1}\right) / 2+\delta}^{2}(\mathcal{A})}\right)
$$

per ogni $u \in W_{t_{1}, s_{2}}^{r}(A)$.

Teniamo ora presente (efr. il teorema 8.7 di [1]) che, se $\varepsilon>0$, qualunque siano i numeri reali $r, s_{1}, s_{2}$, con $r \geqslant 1$ e $s_{1} \leqslant s_{2}$, l'iniezione

$$
W_{s_{1}, s_{1}}^{r}(\Lambda) \hookrightarrow L_{\left(s_{1}+s_{3}\right) / 2-r_{1}-\left(s_{2}-s_{1}\right) / 2+\varepsilon}^{2}(\Lambda)
$$

è compatta.

Pertanto, per un noto risultato dovuto a J. Peetre (cfr. il lemma 3 al n. 8 di [5]), si ha che la (3.6) implica che 1'operatore $u \rightarrow \mathcal{A}^{1} u$, definito in $W_{s_{1} t_{2}}^{r}(\Lambda)$ ed a valori in $E_{r, s_{1}, t_{3}}(A)$, ha immagine chiusa; e ciò, se $s_{1}-r+1$ appartiene a $\Sigma_{1}$ e non è zero reale di $g_{1}$ appartenente a $\{-2 m+2, \ldots, 0\}$, contrastata con il teorema II.

Analogamente si ha che la (3.7) implica che l'operatore $u \rightarrow \mathcal{A}^{2} u$, definito in $W_{t_{1}, s_{2}}^{r}(\Lambda)$ ed a valori in $E_{r, t_{1}, s_{2}}(\Lambda)$, ha immagine chiusa; e ciò, se $s_{2}-r+1$ appartiene a $\Sigma_{2}$ e non è zero reale di $g_{2}$ appartenente a $\{-2 m+2, \ldots, 0\}$, contrastata con il teorema II.

Resta così provato che la (1.14) di [3] non può sussistere con $\varepsilon>0$, quando uno dei due numeri $s_{1}-r+1$ e $s_{2}-r+1$ soddisfa le suddette condizioni, e quindi, per la compattezza dell'iniezione (3.8) e per il citato lemma 3 di [5], si ha allora che $o$ il nucleo di $A_{r, s_{1}, s_{2}}$ non ha dimensione finita oppure l'immagine di $\mathcal{A}_{r, s_{1}, s_{2}}$ non è chiusa. Si ha perciò la tesi. 


\section{BIBLIOGRAFIA}

[1] A. Afantaggiati - M. Troisi, Spazi di Sobolev con peso e problemi ellitici in un angolo, $I$, Ann. di Mat. pura e appl., 95 (1973), pp. 361-408.

[2] A. Avantaggiati - M. Troisi, Spazi di Sobolev con peso e problemi ellittici in un angolo, $I I$, Ann. di mat. pura e appl., 97 (1973), pp. 207-252.

[3] A. Avantaggiati - M. Troisr, Spazi di Sobolev con peso e problemi ellittici in un angolo, III, Ann. di Mat. pura e appl. 99 (1974), pp. 1-51.

[4] I. C. GoHBERG - M. G. KREIN, The basic propositions on defect numbers, root numbers and indices of linear operators (in russo), Uspehi Mat. Nauk, 12 (1957), pp. 43-118; trad. inglese: Amer. Math. Soc. Transl., ser. 2, 13 (1960), pp. 185.264.

[5] J. Peetre, Another approach to elliptio boundary problems, Comm. Pure Appl. Math., 14 (1961), pp. 711-731. 